\title{
Phonon Heat Transport Near the Melting Point
}

\author{
Molecular dynamics simulations can fully describe phonon propagation in aluminum, which \\ could enable accurate predictions of phonon thermal conductivity.
}

\section{by David Andersson*}

$\mathrm{P}$ honons-collective vibrational modes of crystals-influence many physical properties, such as free energy and phase stability, thermal expansion, and thermal conductivity, all of which are important to a wide range of technological applications. Thermal conductivity in particular is an example that requires an accurate account of the full phonon spectra and related phonon lifetimes. Usually, researchers can accurately predict phonon thermal conductivity using a "low-order" perturbation theory, in which the vibrational motion is determined by simple interactions between pairs and triplets of atoms [1]. However, these predictions diverge from experiments at higher temperatures, where higher-order effects like multiatom interactions become increasingly important [2]. To pinpoint which higher-order effects have the largest impact, Albert Glensk from the Max Planck Institute for Iron Research, Germany, and colleagues performed inelastic neutron scattering (INS) experiments on aluminum

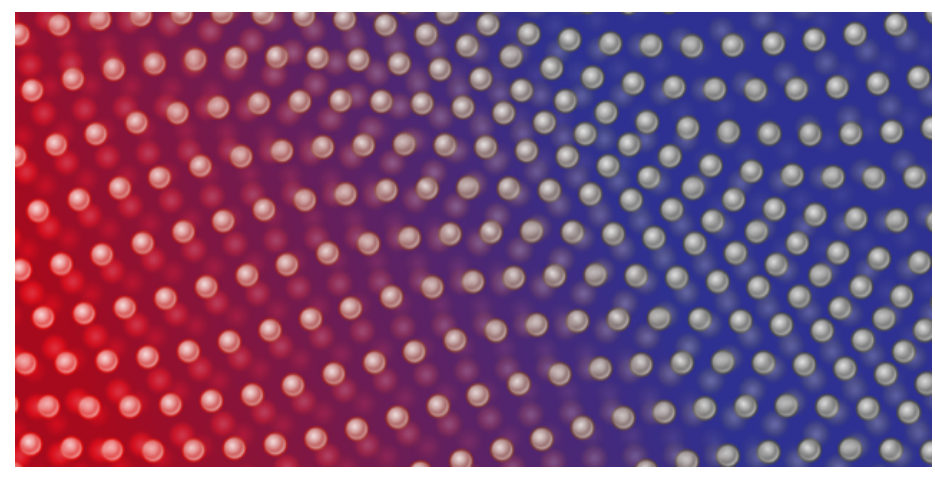

Figure 1: Lattice vibrations (phonons) play an important role in determining material properties, including thermal conductivity. To model the phenomenon accurately and efficiently, the most important factors governing phonon physics need to be identified. (APS/Alan Stonebraker)

* Los Alamos National Laboratory, Los Alamos, NM, USA and compared the observed phonon behavior to theoretical predictions [3]. They showed that low-order perturbation theory failed to predict the phonon lifetimes at temperatures near the melting point, whereas $a b$ initio molecular dynamics (AIMD) simulations, which include higher-order effects, showed quantitative agreement with the experiments. In particular, the team identified anharmonic pair interactions as the dominant higher-order contribution. The result is a very notable milestone in the quest for predictive and quantitative first-principles calculations of material properties.

Thermal conductivity due to phonons is a function of three parameters: the speed at which phonons propagate through the lattice, the phonon heat capacity, and how far phonons can travel before they are scattered by lattice imperfections, electrons, and other phonons. In metals, the contribution that phonons make to thermal conductivity is relatively insignificant, as electrons carry most of the heat. In insulators or semiconductors on the other hand, the electronic component of thermal transport is small, and phonons dominate. Although the study by Glensk and colleagues is devoted to aluminum, in which phonons play a minor role, the basic physics concepts extend to other materials where phonons are key to thermal transport.

At the simplest level, thermal conductivity can be measured by applying a heat source and a sink, monitoring the resulting temperature gradient, and then using Fourier's law to calculate the conductivity's value. However, this technique provides little information about the underlying phonon transport mechanism. Deeper insight comes from INS experiments, which measure how much energy incident neutrons lose when they are scattered by atoms in the sample. This captures the mechanistic aspects of phonon thermal conductivity by revealing what phonon frequencies-or modes-are present as well as the width of each phonon peak [4]. The width of the peak is inversely proportional to the phonon lifetimes: a broad peak indicates significant phonon scattering and short lifetimes and therefore a reduced thermal conductivity from this particular phonon mode. With knowledge of the phonon spectra and lifetimes, the phonon thermal conductivity can be calculated by summation over all modes. If the phonons carry the 
majority of the heat current, the result should be the same as that measured in the simple conductivity experiment described above.

Glensk and colleagues performed INS experiments on aluminum at temperatures around $900 \mathrm{~K}$, which is just below the melting point of $933 \mathrm{~K}$. They derived a phonon spectrum from the INS data and compared it to perturbation theory, which is an approximative model that calculates how nearby atoms react when one atom is displaced from its equilibrium position. The lowest-order response is a harmonic, springlike force between two atoms. Higher-order responses can include multiple atoms and anharmonic forces. By including third-order effects, perturbation theory does quite well at predicting the observed phonon properties in aluminum and other materials, even at high temperatures [5]. A key result by Glensk and colleagues, however, is that the accuracy decreases for temperatures approaching the melting point. In aluminum at such temperatures, even higherorder anharmonicity and multiphonon interactions become increasingly important and must be included for predicted phonon spectra and linewidths to reach quantitative agreement with experiments.

These additional phenomena can be accounted for by expanding the perturbation method still further to include fourth-order effects and higher [6]. Another more direct (though computationally expensive) approach is to use AIMD simulations, which naturally include interactions of higher orders. Glensk and colleagues demonstrate that by including these higher-order interactions that are inherent to AIMD techniques, phonon lifetimes can be reproduced right up to the melting point. Importantly, their AIMD simulations also show that the strongest control on phonon lifetimes in aluminum is anharmonic pair interactions between nearest-neighbor atoms, rather than complex interactions involving multiple sites on the lattice. By revealing which interactions are crucial and which do not need to be calculated, this conclusion provides critical guidance for future studies of high-temperature phonon properties. As an example, Glensk and colleagues demonstrate how it motivates development of accurate and computationally efficient empirical potentials that capture the full high-temperature anharmonicity and thus the phonon lifetimes, but with a much-reduced computational cost compared to AIMD simulations. It is also possible to "skip" the lifetime prediction step and directly calculate the thermal conductivity by incorporating the critical anharmonicity and multiphonon processes in thermodynamic frameworks like the Green-Kubo or direct nonequilibrium methods [7].

Aluminum is a simple metal and the next step would be to extend this methodology to elements with a more complex electronic structure as well as to compounds of more than one element. If this step can be successfully navigated, the findings described by Glensk and colleagues will have far-reaching implications for modeling and simulating phonon-related properties in applications as well as for materials design. There are, of course, additional scattering mechanisms that impact phonon lifetimes, of which, for example, point defects, alloying elements, and grain boundaries are well known and studied. There are also interesting cases where interactions with the electronic and magnetic degrees of freedom may be important, such as in uranium dioxide, whose thermal conductivity is drastically reduced because of phonon-spin interactions [8]. Including these additional contributions in ab initio models of phonon lifetimes and, by extension, thermal conductivity predictions, represents another challenge beyond the accomplishments of Glensk and colleagues to be addressed by the research community.

This research is published in Physical Review Letters.

\section{REFERENCES}

[1] X. Tang, C. W. Li, and B. Fultz, "Anharmonicity-induced phonon broadening in aluminum at high temperatures," Phys. Rev. B 82 , 184301 (2010).

[2] R. A. Cowley, "Anharmonic crystals," Rep. Prog. Phys. 31, 123 (1968).

[3] A. Glensk, B. Grabowski, T. Hickel, J. Neugebauer, J. Neuhaus, K. Hradil, W. Petry, and M. Leitner, "Phonon lifetimes throughout the Brillouin zone at elevated temperatures from experiment and ab initio," Phys. Rev. Lett. 123, 235501 (2019).

[4] J. W. L. Pang, W. J. L. Buyers, A. Chernatynskiy, M. D. Lumsden, B. C. Larson, and S. R. Phillpot, "Phonon lifetime investigation of anharmonicity and thermal conductivity of $\mathrm{UO}_{2}$ by neutron scattering and theory," Phys. Rev. Lett. 110, 157401 (2013).

[5] X. Tang, C. W. Li, and B. Fultz, "Anharmonicity-induced phonon broadening in aluminum at high temperatures," Phys. Rev. B 82, 184301 (2010).

[6] T. Feng, L. Lindsay, and X. Ruan, "Four-phonon scattering significantly reduces intrinsic thermal conductivity of solids," Phys. Rev. B 96, 161201 (2017).

[7] P. K. Schelling, S. R. Phillpot, and P. Keblinski, "Comparison of atomic-level simulation methods for computing thermal conductivity," Phys. Rev. B 65, 144306 (2002).

[8] K. Gofryk et al., "Anisotropic thermal conductivity in uranium dioxide," Nat. Commun. 5, 4551 (2014).

10.1103/Physics.12.135 